OPEN ACCESS

Edited by:

Henry T. Nguyen,

University of Missouri, USA

Reviewed by:

Han Asard,

University of Antwerp, Belgium

Woo Taek Kim

Yonsei University, South Korea

${ }^{*}$ Correspondence:

Byeong-ha Lee

byeongha@sogang.ac.kr

Sanjib Kumar Panda

drskpanda@gmail.com

Specialty section: This article was submitted to

Plant Physiology,

a section of the journal

Frontiers in Plant Science

Received: 03 May 2016 Accepted: 05 October 2016 Published: 21 October 2016

Citation:

Alavilli H, Awasthi JP, Rout GR, Sahoo L, Lee B-h and Panda SK (2016) Overexpression of a Barley Aquaporin Gene, HvPIP2;5 Confers Salt and Osmotic Stress Tolerance in Yeast and Plants.

Front. Plant Sci. 7:1566 doi: 10.3389/fpls.2016.01566

\section{Overexpression of a Barley Aquaporin Gene, HvPIP2;5 Confers Salt and Osmotic Stress Tolerance in Yeast and Plants}

\author{
Hemasundar Alavilli ${ }^{1}$, Jay Prakash Awasthi ${ }^{2}$, Gyana R. Rout ${ }^{3}$, Lingaraj Sahoo ${ }^{4}$, \\ Byeong-ha Lee ${ }^{1 *}$ and Sanjib Kumar Panda ${ }^{2 *}$
}

${ }^{1}$ Department of Life Science, Sogang University, Seoul, Korea, ${ }^{2}$ Plant Molecular Biotechnology Laboratory, Department of Life Science and Bioinformatics, Assam University, Silchar, India, ${ }^{3}$ Department of Agricultural Biotechnology, Orissa University of Agriculture and Technology, Bhubaneswar, India, ${ }^{4}$ Department of Bioscience and Biotechnology, Indian Institute of Technology, Guwahati, India

We characterized an aquaporin gene HvPIP2;5 from Hordeum vulgare and investigated its physiological roles in heterologous expression systems, yeast and Arabidopsis, under high salt and high osmotic stress conditions. In yeast, the expression of HVPIP2;5 enhanced abiotic stress tolerance under high salt and high osmotic conditions. Arabidopsis plants overexpressing HVPIP2;5 also showed better stress tolerance in germination and root growth under high salt and high osmotic stresses than the wild type (WT). HVPIP2;5 overexpressing plants were able to survive and recover after a 3-week drought period unlike the control plants which wilted and died during stress treatment. Indeed, overexpression of HVPIP2;5 caused higher retention of chlorophylls and water under salt and osmotic stresses than did control. We also observed lower accumulation of reactive oxygen species (ROS) and malondialdehyde (MDA), an end-product of lipid peroxidation in HVPIP2;5 overexpressing plants than in WT. These results suggest that HVPIP2;5 overexpression brought about stress tolerance, at least in part, by reducing the secondary oxidative stress caused by salt and osmotic stresses. Consistent with these stress tolerant phenotypes, HvPIP2;5 overexpressing Arabidopsis lines showed higher expression and activities of ROS scavenging enzymes such as catalase (CAT), superoxide dismutase (SOD), glutathione reductase (GR), and ascorbate peroxidase (APX) under salt and osmotic stresses than did WT. In addition, the proline biosynthesis genes, $\Delta^{1}$-Pyrroline-5-Carboxylate Synthase 1 and 2 (P5CS1 and P5CS2) were up-regulated in HvPIP2;5 overexpressing plants under salt and osmotic stresses, which coincided with increased levels of the osmoprotectant proline. Together, these results suggested that HvPIP2;5 overexpression enhanced stress tolerance to high salt and high osmotic stresses by increasing activities and/or expression of ROS scavenging enzymes and osmoprotectant biosynthetic genes.

Keywords: yeast, Arabidopsis, aquaporin, barley, HvPIP2;5, overexpression, stress tolerance 


\section{INTRODUCTION}

Aquaporins belong to major intrinsic proteins (MIPs) that are present from prokaryotes to plants and animals. These proteins facilitate the transport of water and small uncharged molecules across biological membranes (Park and Saier, 1996; Heymann and Engel, 1999; Engel and Stahlberg, 2002; Zardoya et al., 2002; Maurel et al., 2008, 2015). MIPs are categorized into two groups: aquaporins that show water-specific channel activity and glycerol-uptake facilitators (GLPs or aquaglyceroporins) that have channel activity for additional small molecules such as glycerol or urea (Park and Saier, 1996; Heymann and Engel, 1999; Engel and Stahlberg, 2002; Zardoya et al., 2002; Zardoya, 2005; Maurel et al., 2008). In plants, all MIPs, except for GlpF-like intrinsic proteins (GIPs), exhibit water-specific channel activity; therefore, most plant MIPs are collectively called aquaporins (Maurel et al., 2008, 2015). Recently, it has been shown that plant MIPs can transport other small molecules such as $\mathrm{CO}_{2}$ and ammonia (Uehlein et al., 2003; Jahn et al., 2004).

Compared to genomes of other organisms, plant genomes contain a higher number of aquaporins (Wang et al., 2014). For instance, there are 35 aquaporin genes in Arabidopsis thaliana (Maurel, 2007), 22 in Jatropha curcas (Khan et al., 2015), 36 in Zea maize (Chaumont et al., 2001) and over 40 in Hordeum vulgare (Hove et al., 2015) while Escherichia coli (Gomes et al., 2009), Caenorhabditis elegans (Ishibashi et al., 2011), Drosophila melanogaster (Spring et al., 2009), and Homo sapiens (Day et al., 2014) contain 2, 11, 7, and 12, respectively.

The high diversity in plant aquaporins suggests variation of their physiological roles. Indeed, aquaporins were shown to be associated with vital physiological processes such as photosynthesis, nitrogen fixation, nutrient uptake and other environmental stress responses (Li et al., 2014; Hove et al., 2015; Sun et al., 2015). Plant aquaporins are classified into five subgroups, i.e.,: the plasma membrane intrinsic proteins (PIPs), tonoplast intrinsic proteins (TIPs), nodulin26-like intrinsic proteins (NIPs), small basic intrinsic proteins (SIPs) and the recently identified uncategorized (X) intrinsic proteins (XIP) (Maurel et al., 2015). Based on sequence divergence, PIPs are further divided into PIP1 and PIP2 subclasses each consisting of several isoforms which play important roles in determining hydraulic conductivity particularly in roots (Martre et al., 2002; Siefritz et al., 2002; Javot et al., 2003; Postaire et al., 2010). Analyses on PIP1 and PIP2 from barley and maize revealed that the PIP2 proteins had higher water transport activity than PIP1 proteins in Xenopus oocytes (Chaumont et al., 2000; Horie et al., 2011). When PIP2 was co-expressed with functional or even nonfunctional PIP1 proteins, water transport activity of PIP2 was enhanced (Chaumont et al., 2000; Fetter et al., 2004; Horie et al., 2011). This enhanced water transport was attributed to their ability to form heterotetramers for proper trafficking to the plasma membrane (Fetter et al., 2004; Zelazny et al., 2007).

Dynamic changes in the expression levels of many PIP genes were observed in response to drought stress, suggesting their involvement in stress responses by regulating water balance (Afzal et al., 2016). Studies with PIP-defective mutants or overexpressing plants also linked the roles of PIP proteins to water-deficit stress tolerance. For example, when expression of a tobacco PIP1 member (NtAQP1) was reduced by antisense technology, the NtAQP1-downregulated tobacco plants showed reduced root hydraulic conductivity and wilting phenotypes under water stress (Siefritz et al., 2002). Physcomitrella patens PIP2;1 or PIP2;2 knockouts showed low water permeability with drought-sensitive phenotypes (Lienard et al., 2008). Reduction in water permeability of protoplasts and root hydraulic conductivity were observed respectively in Arabidopsis PIP1;2 and PIP2;2defective mutants but without clear developmental defects (Kaldenhoff et al., 1998; Javot et al., 2003). Overexpression of several PIP genes from various plants including Oryza sativa, Vicia faba, Nicotiana tabacum, and Triticum aestivum successfully enhanced water stress tolerance in transgenic plants (Lian et al., 2004; Cui et al., 2008; Sade et al., 2010; Zhou et al., 2012). Interestingly, some contrasting results (i.e., stress sensitive phenotypes in PIP overexpressing plants) have also been reported, implying the complexity of PIP function in plants (Aharon et al., 2003; Katsuhara et al., 2003; Jang et al., 2007; Li et al., 2015).

Barley (Hordeum vulgare L.) is one of the most agronomically cultivated crops; it is more adaptable to drought, salinity and cold than other cereal crops (Katsuhara et al., 2014; Hove et al., 2015). These characteristics would possibly make the barley gene pool, including barley aquaporins, as one of stress-adaptive genetic resources. Although, several PIPs have been identified in barley, only few of them have been functionally characterized thus far.

In this study, we overexpressed barley PIP2;5 (HvPIP2;5) in yeast and Arabidopsis and characterized these lines to understand the functions of the barley PIP gene under high salt and high osmotic stress conditions.

\section{MATERIALS AND METHODS}

\section{HvPIP2;5 Expression Vector Construction}

Barley (Hordeum vulgare cv. NP21) cDNA was prepared using superscriptTM III reverse transcriptase (Invitrogen, USA), and total RNA was extracted with TRIzol ${ }^{\circledR}$ Reagent (Ambion, USA). A 873 bp-length HvPIP2;5 coding sequence (GenBank Accession number: AB377270.1) was cloned into TA cloning vector pTOPO2.1 (Invitrogen, Carlsbad, CA, USA) using gene specific primers (Supplementary Table 1). The coding sequences of $H v P I P 2 ; 5$ was cloned into yeast expression vector pYES2.0 (Invitrogen, USA) at the EcoRI site and named pYES2: HvPIP2;5. For plant transformation, $H v P I P 2 ; 5$ coding sequence was cloned into a standard plant binary vector pCAMBIA2301. The resulting overexpression construct was named pCAMBIA230135S:HvPIP2;5.

\section{Transformation of Yeast and Stress Analysis}

The HvPIP2;5 coding sequence under control of GAL1 promoter in pYES2 yeast expression vector was introduced into yeast FY3 cells. As controls, FY3 cells containing pYES2 vector only (vector control) and FY3 strain only were used in stress assays. The yeast strain FY3 was transformed with a pYES2 empty vector or pYES2:HvPIP2;5 recombinant vector by lithium acetate method 
(Kawai et al., 2010) and selected on SC medium devoid of uracil. Yeast cells expressing HvPIP2;5 along with control cells were grown on YPD solid medium (1\% Yeast extract, $2 \%$ peptone, and $2 \%$ glucose).

For stress analysis, transformed yeast cells were propagated in SC-U medium containing $2 \%$ galactose for $12 \mathrm{~h}$ and cell density was adjusted to 1.0 of OD600 followed by serial dilutions. Yeast cell were spotted on YPD medium supplemented with PEG (4\%) or $\mathrm{NaCl}(200 \mathrm{mM})$, respectively. Plates were maintained at $30^{\circ} \mathrm{C}$ and growth was monitored after 2 days.

\section{Generation of HvPIP2;5 Overexpressing Arabidopsis Lines and Stress Analysis}

Using Agrobacterium tumefaciens GV3101 harboring pCAMBIA2301-35S:HvPIP2;5, Arabidopsis Columbia-0 plants were transformed via floral dipping method (Clough and Bent, 1998). Homozygote for 35S:HvPIP2;5 insertion was selected at T4 generation by analyzing kanamycin resistance at each generation.

For germination analysis, the seeds of WT and homozygote HvPIP2;5 OE Arabidopsis lines were plated on half strength Murashige Skoog (MS) media and allowed to germinate at $22^{\circ} \mathrm{C}$ and $60 \%$ relative humidity with a $16 / 8 \mathrm{~h}$ light-dark photo cycle after 3 day stratification at $4^{\circ} \mathrm{C}$.

Final concentration of $100-200 \mathrm{mM} \mathrm{NaCl}$ (for high salt stress) or $10-20 \%$ PEG (for high osmotic stress) was supplemented to the MS media for stress administration. Hereby salt stress or osmotic stress indicates high salt stress or high osmotic stress, respectively. Emergence of cotyledons was used as a germination criterium. The number of germinated seeds was expressed as a percentage of total number of seeds planted after 7 days. For root elongation analysis, vertically grown seedlings with $1-1.5 \mathrm{~cm}$ long root were transferred onto a MS vertical plate supplemented with or without stress agents (200 mM NaCl and 20\% PEG). Root length was measured 15 days after transfer. Root length of seedlings under stress conditions was expressed as a percentage of their respective controls grown on normal MS medium. All stress analysis experiments were conducted three times and each contained 3 biological repeats. For drought test with soil-grown plants, 4 week old seedlings were subjected to drought stress by withholding water supply for 21 days and then re-watered. For salt and osmotic stress, 3 week old plants were irrigated with either half strength MS liquid (control) or half strength MS liquid supplemented either with $200 \mathrm{mM} \mathrm{NaCl}$ or $20 \%$ PEG to impose stress for 15 days at 2 day intervals and then harvested for analysis. The chlorophyll, proline, and malondialdehyde (MDA) content in control or stress treated plants were determined by the methods reported previously by Lichtenthaler (1987), Bates et al. (1973), and Heath and Packer (1968), respectively.

\section{Water Loss and Relative Water Content Analysis}

Rosette leaves of 3 week old seedlings were detached, weighed and placed on paper under the fume hood to administrate drought stress. Fresh weights of rosette leaves were measured each hour for $5 \mathrm{~h}$. Water loss was calculated as the loss in fresh weight of the samples. For relative water content analysis, the root from WT and the HvPIP2;5 OE lines were excised and treated either with $200 \mathrm{mM} \mathrm{NaCl}$ or $20 \%$ PEG or deionized water after measuring the fresh weight. After $24 \mathrm{~h}$ of incubation, the tissues were weighed again for their turgid weight and then dried completely to obtain dry weight. The root RWC was calculated by the following formula; RWC (\%) $=[$ (fresh weight - dry weight $) /($ turgid weight - dry weight $)] \times 100($ Weatherley, 1950).

\section{Reactive Oxygen Species Analysis and Antioxidant Enzyme Activity Assay}

In situ detection of superoxide by nitro blue tetrazolium (NBT) staining and hydrogen peroxide by 3, 3'diaminobenzidine (DAB) staining was performed according to methods previously described by Rao and Davis (1999) and Ramel et al. (2009). For this, 3 week old seedlings were grown on MS medium with 0, $100,200 \mathrm{mM} \mathrm{NaCl}$ and the third leaf from the top was used. Superoxide radicals were visualized as blue color produced by NBT precipitation while hydrogen peroxide spots were visualized as brown color due to DAB polymerization.

For ROS quantification and antioxidant enzyme activity assay, 3 week old plants were irrigated with either half strength MS liquid (control) or half strength MS liquid supplemented either with $200 \mathrm{mM} \mathrm{NaCl}$ or $20 \%$ PEG to impose stress for 15 days at 2 day intervals and then harvested for analysis. Contents of superoxide and hydrogen peroxide were estimated by methods previously described by Elstner and Heupel (1976) and Sagisaka (1976), respectively. For antioxidant enzyme assay, the samples ( $200 \mathrm{mg}$ tissue) were homogenized in $1.5 \mathrm{~mL}$ of $0.1 \mathrm{M}$ phosphate buffer ( $\mathrm{pH}$ 6.8) containing $1 \mathrm{mM}$ ethylenediamine tetra-acetic acid (EDTA) and 1\% polyvinylpyrrolidone (PVP) in a chilled pestle and mortar. The homogenate was then centrifuged at $17,000 \mathrm{~g}$ for $15 \mathrm{~min}$ at $4^{\circ} \mathrm{C}$. The supernatant was quantified by the Bradford method (Bradford, 1976) and used for the assay of catalase (CAT), superoxide dismutase (SOD) and glutathione reductase (GR) (Chance and Maehly, 1955; Smith et al., 1988; Gupta et al., 1993). For the ascorbate peroxidase (APX) activity assay, a final concentration of $1 \mathrm{mM}$ ascorbic acid was added to the assay buffer (Nakano and Asada, 1981).

\section{Gene Expression Analysis}

Plants were treated with water for control or $300 \mathrm{mM} \mathrm{NaCl}$ for $6 \mathrm{~h}$ or 20\% PEG for $6 \mathrm{~h}$, and total RNAs were extracted using RNeasy Plant mini Kit (Qiagen, Germany). cDNA was synthesized using RevertAid First Strand cDNA Synthesis Kit (ThermoFisher, USA), and resulting cDNAs were used for semi-quantitative RT or qRT-PCR experiments for selected genes. Primer pairs used in experiments are shown in Supplementary Table 1.

\section{Statistical Analysis}

All statistical comparisons between variances were determined by ANOVA (Analysis of variance) and least significant differences (LSD) between variants were calculated using Statistix 8.1 computation software. Statistically significant mean values were denoted as $*(P \leq 0.05)$. 


\section{RESULTS}

\section{Sequence Analysis of HvPIP2;5}

Previously, we identified several barley plasma membrane intrinsic protein $(H v P I P)$ genes and examined their expressions under salt and osmotic stress conditions (Horie et al., 2011; Katsuhara et al., 2014). For further functional and physiological characterization, we selected HvPIP2;5 (Genbank \#AB377270), one of the HvPIP2 genes that possesses abundant transcripts and demonstrates down-regulation of gene expression under salt and osmotic stresses (Horie et al., 2011; Katsuhara et al., 2014). The HvPIP2;5 gene encodes a polypeptide of 291 amino acids with an estimated molecular mass of $30.3 \mathrm{KDa}$ and an isoelectric point of 8.28 as predicted by ExPaSy bioinformatics tools for protein structure analysis (http://web.expasy.org/ compute_pi/). HvPIP2;5 protein shares $81 \%$ identity with Arabidopsis PIP2;5 protein (AtPIP2;5, AT3G54820). As shown in Supplementary Figure 1A, both HvPIP2;5 and AtPIP2;5 harbor two Asn-Pro-Ala (NPA) motifs in addition to the highly conserved amino acid sequence "HINPAVTFG" which is reportedly conserved among all the MIP superfamily proteins ( $\mathrm{Li}$ et al., 2009; Zhou et al., 2012). Conserved phosphorylation-target serine residues were also found in the C-termini of both PIP2;5 orthologs (Supplementary Figure 1A; Hove et al., 2015). The predicted HvPIP2;5 protein contained aquaporin-characteristic six transmembrane spanning $\alpha$-helices $(\mathrm{H} 1-\mathrm{H} 6)$ with presence of $35.17 \%$ alpha helix, $30 \%$ random coil, $24.48 \%$ random coil, and 10.34\% beta turn (Supplementary Figures 1B,C).

It has been recently reported that the barley genome comprises at least 40 aquaporin genes with 5 PIP1 genes and 9 PIP2 genes (Hove et al., 2015). Multiple sequence alignment using all reported HvPIP2 isoforms was performed using Mega 6 software (http://www.megasoftware.net). As expected, HvPIP2 proteins showed high degree of homology among them, and HvPIP2;5 was located in the same clade as HvPIP2;1 with $85 \%$ identity to HvPIP2;1 (Supplementary Figure 1D).

\section{Increased Tolerance of HvPIP2;5-Expressing Yeast under High Salt and Osmotic Stresses}

A yeast expression system was employed to examine the functions of the HvPIP2;5 gene under high saline and high osmotic conditions. As shown in Figure 1A, yeast strains transformed with HvPIP2;5 or empty vector and FY3 cells could grow up to $10^{-6}$ dilution on YPD plates. In the

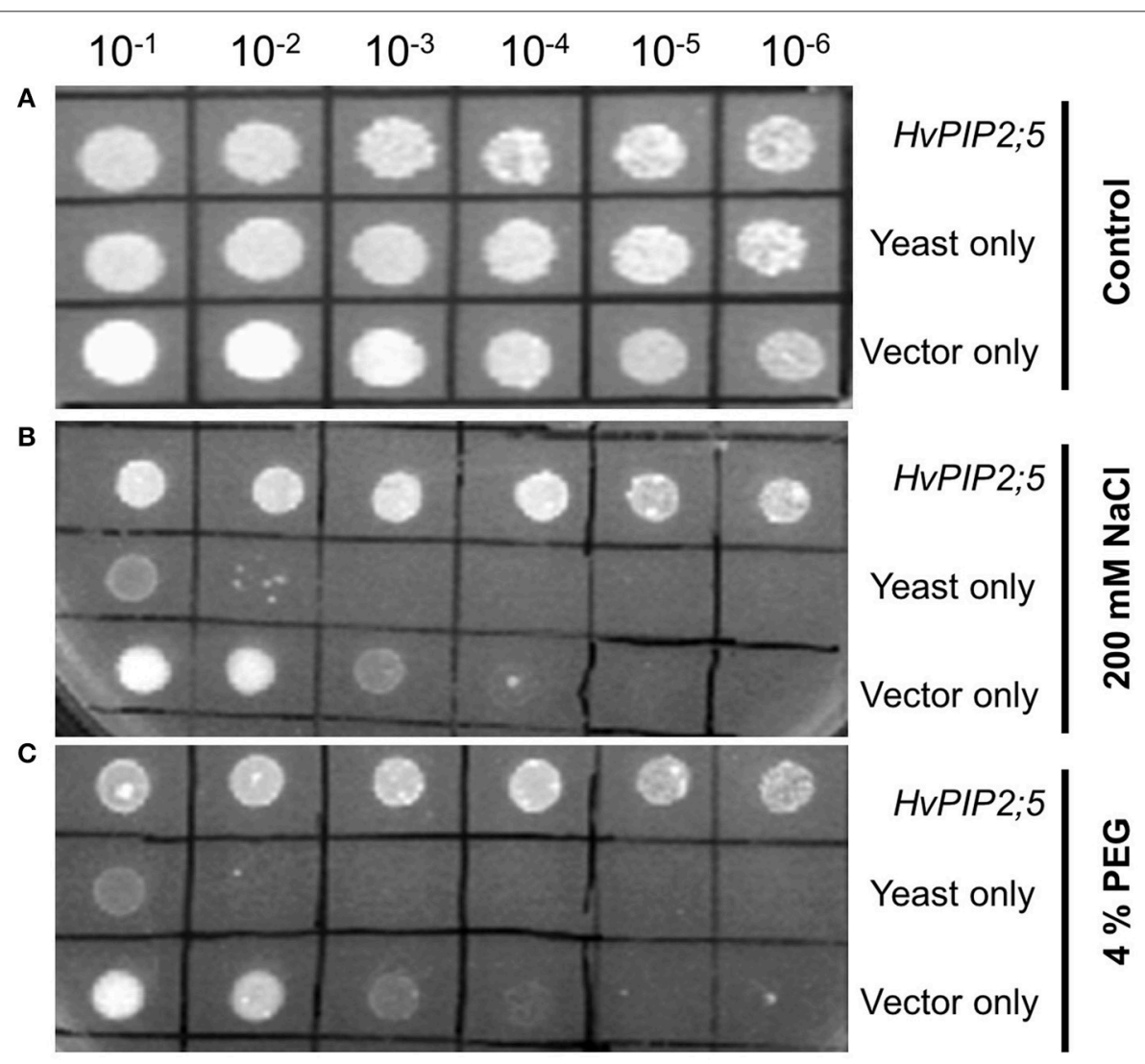

FIGURE 1 | Effect of HvPIP2;5 expression in yeast under salt and osmotic stresses. Yeast cells harboring the HVPIP2;5 expressing construct (HVPIP2;5), yeast cells only (yeast), and yeast cells with the vector pYES2 only (vector only) were subjected to $200 \mathrm{mM} \mathrm{NaCl}$ and $4 \%$ PEG. Cell density was adjusted to OD-600 at 1.0 and serial dilutions were made at each step. Ten microliter of each dilution was spotted on (A) YPD plates without stress. (B) YPD plates supplemented with 200 mM $\mathrm{NaCl}$. (C) Supplemented with 4\% PEG. Photographs were taken after $48 \mathrm{~h}$ of incubation at $30^{\circ} \mathrm{C}$. 
presence of $200 \mathrm{mM} \mathrm{NaCl}$, pYES2 vector-containing FY3 cells grew only until $10^{-3}$ dilution, whereas the yeast strain transformed with HvPIP2;5 was able to grow until $10^{-6}$ dilution (Figure 1B). Similarly, empty vector containing cells grew only to $10^{-3}$ dilution on the YPD medium supplemented with $4 \%$ polyethylene glycol (PEG), while yeast cells with HvPIP2;5 displayed growth until $10^{-6}$ dilution under the same condition (Figure 1C). Taken together, these results showed that HvPIP2;5 expression in yeast resulted in increased stress tolerance under high salt and high osmotic conditions.

\section{Enhanced Stress Tolerance of HvPIP2;5-Overexpressing Arabidopsis}

To investigate the functional roles of HvPIP2;5 in planta, we expressed $H v P I P 2 ; 5$ in Arabidopsis under the control of CaMV 35S promoter (Supplementary Figure 2A). Two independent homozygous $H v P I P 2 ; 5$ overexpressing (OE) lines were selected. PCR analysis confirmed the presence of the HvPIP2;5 gene in transgenic plants (data not shown) and semi quantitative RT-PCR for $H v P I P 2 ; 5$ expression analysis demonstrated that transgenic lines were overexpressing HvPIP2;5 gene (Supplementary Figure 2B).

Germination under $200 \mathrm{mM} \mathrm{NaCl}$ (high salt stress) and $20 \%$ PEG (high osmotic stress) was first tested for two independent HvPIP2;5 OE lines along with WT. On the control medium, all lines germinated successfully after 7 days of planting. On the medium supplemented with $200 \mathrm{mM} \mathrm{NaCl}$, WT germination ratios were decreased to about 50\%, whereas $H v P I P 2 ; 5 \mathrm{OE}$ lines displayed a $59-60 \%$ germination ratio on salt medium (Figures 2A,D). Also, HvPIP2;5 OE lines displayed a $41-44 \%$ germination ratio in MS medium with 20\% PEG whereas WT germination was reduced to $18 \%$ (Figures 2A,D).

Root growth under salt and osmotic stress conditions was also compared. In root growth assays, we observed increased tolerance of $H v P I P 2 ; 5$ OE lines under salinity and osmotic stresses compared to WT. The relative root length of HvPIP2;5 OE lines was significantly higher in the presence of $200 \mathrm{mM} \mathrm{NaCl}$ (59-62\% vs. $34 \%$ ) or $20 \%$ PEG ( $47-50 \%$ vs. $28 \%$ ) compared to that of WT (Figures 2B,E).

We further evaluated growth performance of WT and HvPIP2;5 OE lines during drought stress. WT and HvPIP2;5 OE plants were grown in well-watered conditions for 4 weeks and then subjected to drought conditions. After 21 days of water withdrawal, WT lines became wilted with retarded growth, whereas HvPIP2;5 OE lines did not wilt as severely as WT. Upon re-watering, WT plants were so severely damaged that they were unable to resume growth. In contrast, the transgenic lines were able to recover and retained survival upon rehydration (Figure 2C).

Chlorophyll degradation is among the changes caused by salt and osmotic stresses. No remarkable differences were observed between WT and HvPIP2;5 OE lines grown under control conditions; however, after stress treatments with 200 $\mathrm{mM} \mathrm{NaCl}$ or 20\% PEG, HvPIP2;5 OE lines showed less chlorophyll degradation when compared with WT. Measurement of chlorophyll contents of stress-treated plants confirmed the better retention of chlorophyll $\mathrm{A}$ in $\mathrm{OE}$ lines than in WT (Figure 2F). WT under salinity stress with $200 \mathrm{mM} \mathrm{NaCl}$ contained only $23 \%$ of chlorophyll A levels under normal conditions, while OE lines under salt stress had largely higher levels of chlorophyll A retention (27-43\%). Additionally, osmotic stress with 20\% PEG caused 51 and 25-31\% chlorophyll A loss in WT and HvPIP2;5 OE, respectively (Figure 2F).

\section{Low Water Loss and High Water Retention in HvPIP2;5 Overexpressing Lines under Stresses}

Dehydration tolerance in HvPIP2;5 lines was assessed by measuring the water loss percentage. For this, rosette leaves from WT and HvPIP2;5 OE lines were detached, placed on paper for dehydration and fresh weight measured. After $5 \mathrm{~h}$ of dehydration, the leaves of WT plants lost $\sim 45 \%$ of their original fresh weight, whereas leaves of the HvPIP2;5 OE lines lost only about 30-35\% from their initial fresh weight (Figure 3A).

In addition, relative water content (RWC) was measured from the root tissues of WT and HvPIP2;5 OE lines under salt or osmotic stress. Under salt stress with $200 \mathrm{mM} \mathrm{NaCl}$, WT lines held 68.1\% RWC, whereas HvPIP2;5 OE lines kept 75-77\% RWC (Figure 3B). Likewise, OE lines contained largely higher RWC than did WT under osmotic stress with 20\% PEG (Figure 3B).

\section{Reduction of Oxidative Stress in HvPIP2;5 Overexpressing Lines under stresses}

Reactive oxygen species (ROS) are generated in plants under drought, salt, and temperature stresses. Using nitro blue tetrazolium (NBT) staining for superoxide and diaminobenzidine (DAB) staining for hydrogen peroxide, we measured the levels of ROS in WT and HvPIP2;5 OE lines under high salt $(200 \mathrm{mM} \mathrm{NaCl})$ and high osmotic stress (20\% PEG). Compared to WT, the HvPIP2;5 OE lines showed significantly weaker NBT staining and less $\mathrm{O}_{2}^{-}$amount under osmotic or salt stress conditions (Figures 4A,B). Similarly, DAB staining and quantification of $\mathrm{H}_{2} \mathrm{O}_{2}$ content revealed lower amounts of $\mathrm{H}_{2} \mathrm{O}_{2}$ in the $\mathrm{OE}$ lines than in WT after $\mathrm{NaCl}$ treatment (Figures 4C,D). However, HvPIP2;5 OE lines did not seem to display very significant reduction in $\mathrm{H}_{2} \mathrm{O}_{2}$ levels after PEG treatment when compared to WT.

Malondialdehyde (MDA) is an end product of lipid peroxidation of cell membrane lipids and a good indicator of oxidative damage (Diao et al., 2011). After salt stress (200 $\mathrm{mM} \mathrm{NaCl}$ ) and osmotic stress (20\% PEG), MDA content was significantly lower in $H v P I P 2 ; 5 \mathrm{OE}$ lines than in WT, which implicates lesser membrane damage in $H v P I P 2 ; 5$ OE lines (Figure 4E). These results suggested that enhanced stress tolerance in $H v P I P 2 ; 5 \mathrm{OE}$ lines might be due, at least in part, to reduced levels of oxidative stress caused by salt and osmotic stresses.

\section{Increased Activities of ROS Scavenging Enzymes in HvPIP2;5 Overexpressing Lines}

To assess the contribution of ROS scavenging enzymes in reduction of oxidative stress caused by salt and osmotic stresses, 

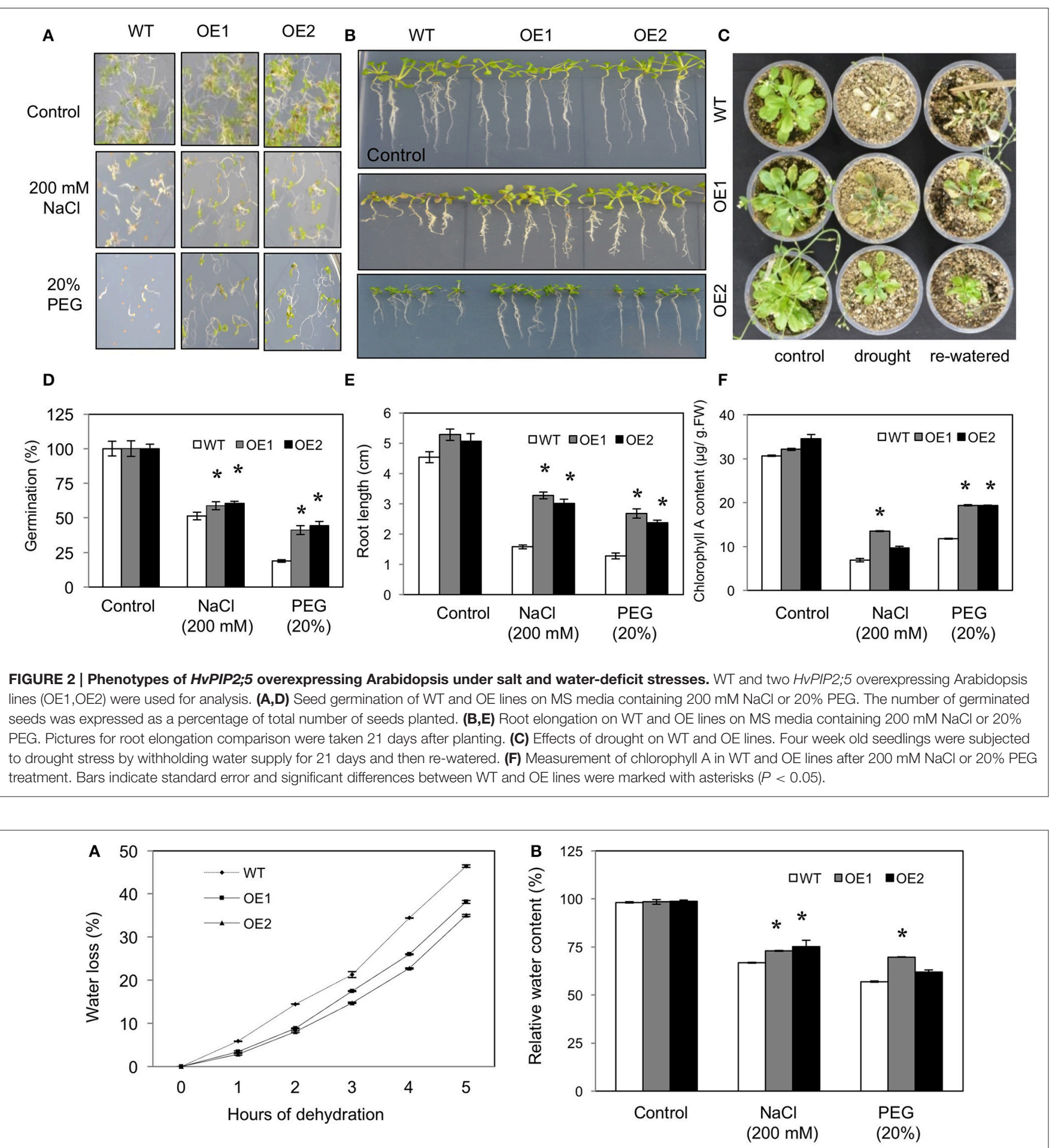

FIGURE 3 | Water loss and retention in HvPIP2;5 overexpressing Arabidopsis under water stress. (A) Comparison of water loss from detached rosette leaves of WT and OE lines, control plants. Water loss was calculated from the loss in fresh weight of the samples. (B) Relative water content estimation in roots of WT and OE lines after $200 \mathrm{mM} \mathrm{NaCl}$ or 20\% PEG treatment. Bars indicate standard error and significant differences between WT and OE lines were marked with asterisks $(P<0.05)$

we measured the activities of catalase (CAT) and superoxide dismutase (SOD) in WT and HvPIP2;5 OE lines under salt and osmotic stress conditions. Although, CAT activity was elevated in both WT and HvPIP2;5 OE lines, consistent with increase of osmotic and saline stresses (Figure 5A), the level of increase of CAT activity was significantly higher in HvPIP2;5 OE lines 


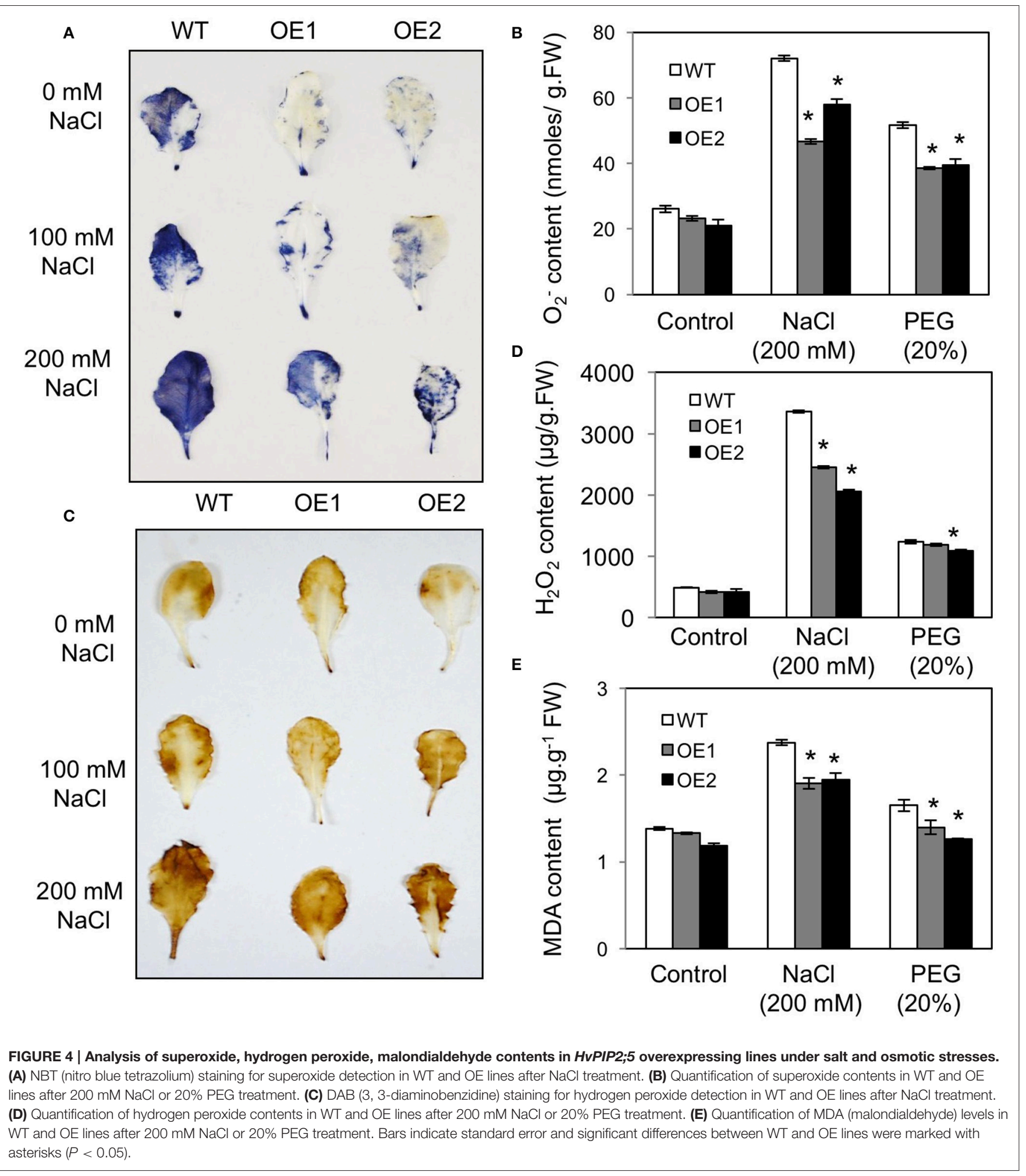

than in WT. Patterns largely similar to those found in the CAT activity assay were observed in SOD activity assay. SOD activity in $H v P I P 2 ; 5$ OE lines were higher than those in WT under salt and osmotic stresses (Figure 5B). Interestingly, both CAT and
SOD activities in HvPIP2;5 OE lines were generally higher than those of WT even under normal conditions.

In addition, we measured the activities of glutathione reductase (GR) and ascorbate peroxidase (APX) in $H v P I P 2 ; 5 \mathrm{OE}$ 

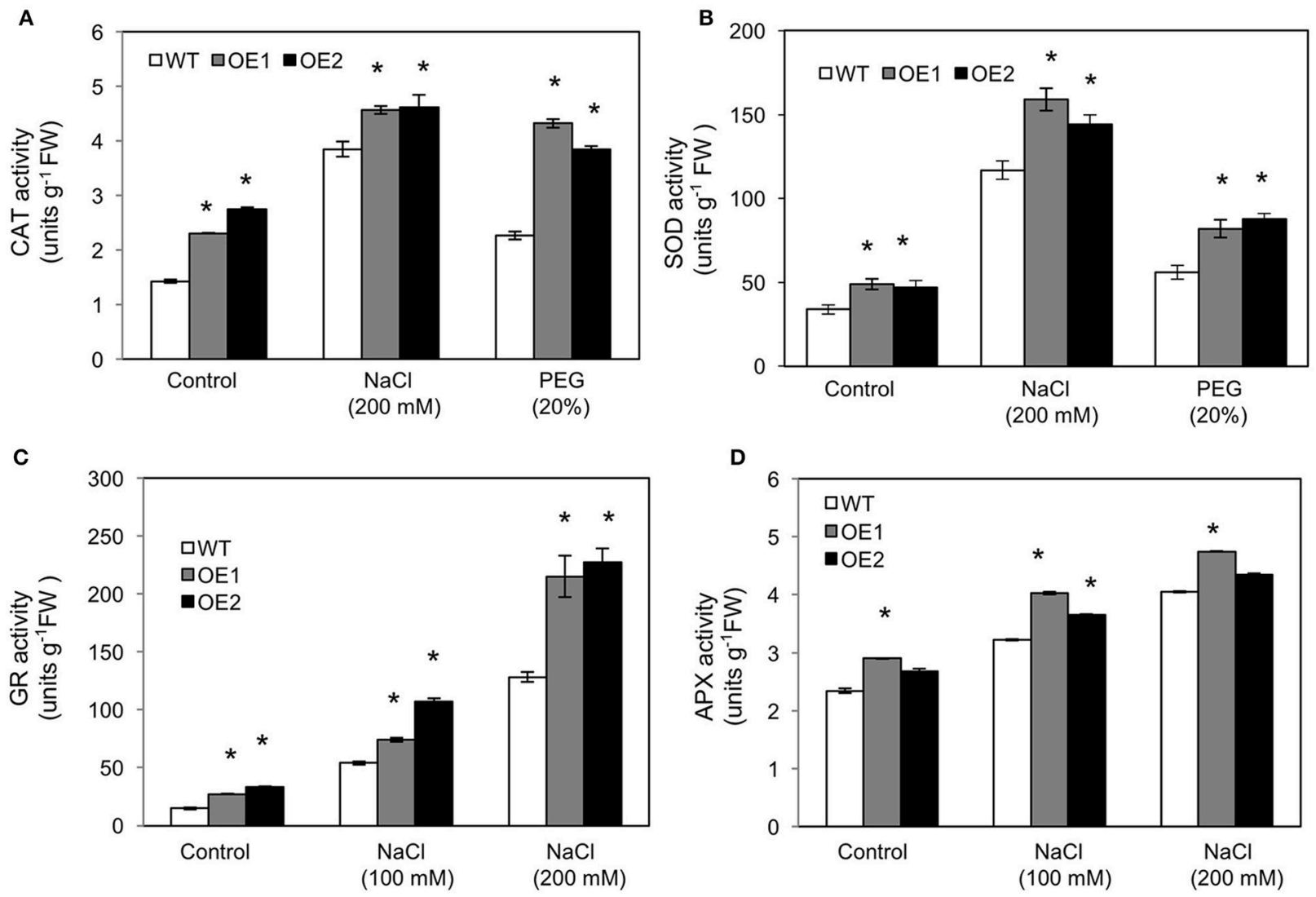

FIGURE 5 | Activities of reactive oxygen species scavenging enzymes in HvPIP2;5 overexpressing lines under salt and osmotic stresses. (A) Activities of catalase (CAT) in WT and OE lines after $200 \mathrm{mM} \mathrm{NaCl}$ or 20\% PEG treatment. (B) Activities of superoxide dismutase (SOD) in WT and OE lines after 200 mM NaCl or 20\% PEG treatment. (C) Activities of glutathione reductase after $\mathrm{NaCl}$ treatments in WT and OE lines. (D) Activities of ascorbate peroxidase after NaCl treatments in WT and OE lines. Bars indicate standard error and significant differences between WT and OE lines were marked with asterisks $(P<0.05)$.

lines under 100 and $200 \mathrm{mM} \mathrm{NaCl}$ conditions. GR and APX are major enzymes for the ascorbate-glutathione cycle which is an important component of the ROS scavenging system in plants (Pang and Wang, 2010). Generally, elevated activities of APX and GR have been shown to correlate with increased salt tolerance in plants (Pang and Wang, 2010). The activities of GR were higher in HvPIP2;5 OE lines than in WT under both normal and salt conditions (Figure 5C). The activities of APX were also largely higher in HvPIP2;5 OE lines than in WT under normal and salt conditions (Figure 5D).

Taken together, these results indicated that reduction of oxidative stress in HvPIP2;5 OE lines under salt and osmotic stresses is possibly related to enhanced activities of ROS scavenging enzymes.

\section{Increased Levels of Proline in HvPIP2;5 Overexpressing Lines}

The amino acid proline acts as an osmolytes and an antioxidant, and high levels of proline enhance stress adaptation under unfavorable conditions (Bates et al., 1973; Hayat et al., 2012). Thus, we investigated expression patterns of the Arabidopsis proline biosynthesis genes, $\Delta^{1}$-Pyrroline-5-Carboxylate Synthase
1 and 2 (P5CS1 and P5CS2) in WT and HvPIP2;5 OE lines. Real time PCR analysis revealed that gene expression levels of P5CS1 and P5CS2 were higher in HvPIP2;5 OE lines than in WT under salt and osmotic stresses (Figures 6A,B). We also measured proline levels in WT and HvPIP2;5 OE lines and found that even under normal conditions, HvPIP2;5 OE lines demonstrated slightly higher proline levels than WT (Figure 6C). After salt treatment, proline levels were increased in both WT and HvPIP2;5 OE lines with much higher increase in OE lines than in WT (Figure 6C). Interestingly, our PEG treatment did not seem to induce proline accumulation. Still, proline levels remained higher in the HvPIP2;5 OE lines than in WT (Figure 6C). This salt-induced proline accumulation correlated with the up-regulation of P5CS1 and P5CS2 expression in HvPIP2;5 OE lines, suggesting a possible molecular mechanism behind the enhanced stress tolerance in HvPIP2;5 OE lines.

\section{DISCUSSION}

Water-deficit stress such as salt and osmotic stress and impede plant growth and development by affecting plant water balance. Aquaporins play important roles as water channels in regulating 

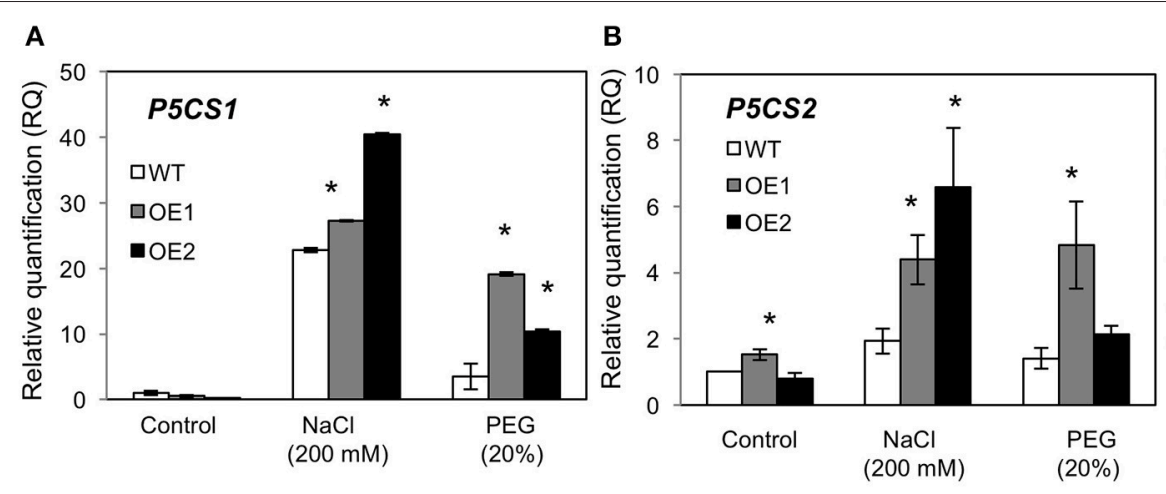

c

FIGURE 6 | Analysis of P5CS1 and P5CS2 gene expression and proline levels in HvPIP2;5 overexpressing lines under salt and osmotic stresses. The expression levels of (A,B) P5CS1 and P5CS2 in WT and OE lines were estimated by quantitative real time PCR. Arabidopsis clatharin gene was used as an internal control for normalization. The expression levels of each gene in WT control (calibrator) were assumed as 1. Three biological replicates were averaged and bars indicate standard error. (C) Proline contents in WT and OE were quantified with three biological replicates. Bars indicate standard error and significant differences between WT and $\mathrm{OE}$ lines were marked with asterisks $(P<0.05)$.

plant water status; thus, many aquaporin genes from diverse plant species have been used in transgenic research to improve waterdeficit stress tolerance in plants (Martre et al., 2002; Cui et al., 2008; Khan et al., 2015).

We found that overexpression of barley PIP2;5 (HvPIP2;5) in both yeast and Arabidopsis improved tolerance to high salt and high osmotic stresses (Figures 1-3). These improved tolerances in HvPIP2;5 overexpressing Arabidopsis were also observed in milder stress conditions $(100 \mathrm{mM} \mathrm{NaCl}$ and $10 \%$ PEG) (Supplementary Figures 3, 4). Increased stress tolerance in HvPIP2;5 overexpressing Arabidopsis was correlated with lower levels of stress-induced ROS (Figure 4 and Supplementary Figure 5), high activity of ROS scavenging enzymes (Figure 5), higher induction of proline biosynthetic gene expression and high levels of osmoprotectant proline (Figure 6). In particular, HvPIP2;5 overexpressing Arabidopsis displayed lower water loss in shoots and higher relative water contents in roots than did WT under salt and osmotic stresses.

While HvPIP2;5 overexpression improved water-stress tolerance in both yeast and Arabidopsis, Arabidopsis PIP2;5 (AtPIP2;5) overexpression brought about reduced osmotic stress tolerance in Arabidopsis and tobacco (Jang et al., 2007). These seemingly contradicting results are not uncommon. In fact, many aquaporin overexpression studies have produced contrasting results-aquaporin-overexpressing plants have shown either positive or negative effects on stress tolerance (Maurel et al., 2008; Martinez-Ballesta and Carvajal, 2014; Zhou et al., 2014). Even overexpression of aquaporins with high homology has resulted in different sensitivities to dehydration stress. For example, Arabidopsis AtPIP $1 ; 2$, rice $R W C 3$, and tobacco $N t A Q P 1$ share approximately $80 \%$ sequence identity. Despite this, overexpression of AtPIP1;2 in tobacco has caused reduced stress tolerance (Aharon et al., 2003) while $R W C 3$ overexpression in rice and $N t A Q P 1$ overexpression in tomato have shown enhanced tolerance under drought and salt stress, respectively (Lian et al., 2004; Sade et al., 2010). In addition, Arabidopsis pip2;2 mutants display defects in hydraulic conductivity despite the expression of a very close homolog AtPIP2;3 which shares $>96 \%$ homology, demonstrating that close aquaporin homologs could not function redundantly even within the same plant (Javot et al., 2003).

Although, HvPIP2;5 and AtPIP2;5 share high sequence homology, there are differences in gene regulation. AtPIP2;5 expression levels remain low in Arabidopsis and are only upregulated by drought and cold (Jang et al., 2007). However, HvPIP2;5 is one of the highly expressed PIPs in barley which is down-regulated by osmotic stress (Katsuhara et al., 2014). These different patterns of gene expression might indicate the divergent functions of PIP2;5 in Arabidopsis and barley, and may be attributed to contrasting stress responses in $H v P I P 2 ; 5$ and AtPIP2;5 overexpressors.

Another explanation for the contrasting results might lie in the difference in protein sequence between HvPIP2;5 and AtPIP2;5 proteins. Amino acid sequence differences are mainly found in the $\mathrm{N}$-terminus which is expected to be exposed on the cytosol side (Walz et al., 1997; Supplementary Figures 1A, 6). Thus, it is tempting to speculate that contrasting stress phenotypes may be due to differences in the N-termini of PIP2;5 proteins which may contain important motifs such as for activity regulation, protein stability, protein interaction, or even subcellular localization. Although transcriptional control of aquaporins appeared to be important for physiological functions (Alexandersson et al., 2005; Guo et al., 2006; Jiang et al., 2016), aquaporin activity is also post-translationally regulated by protein modification including phosphorylation (Johansson et al., 1998; Santoni et al., 2003; Daniels and Yeager, 2005). We have found some differences in phosphorylation sites at the $\mathrm{N}$-terminus of two PIP2;5 proteins (Supplementary Figure 6) which might be important for PIP2;5 function. This differential protein modification might cause variable functional activities of PIP2;5 in plant tissue, particularly where proteins are ectopically expressed due to constant promoter activity. It is interesting to note that Arabidopsis PIP2;1, an AtPIP2;5 close homolog was shown to be a drought response-negative regulator which 
is a target of ubiquitination and degradation by a RING membrane-anchor 1 E3 ubiquitin ligase (Lee et al., 2009). Thus, it might be possible that this kind of Arabidopsis regulation system might function differently on the endogenous PIP2;5 (AtPIP2;5) and the heterologous PIP2;5 (HvPIP2;5) due to the different amino acid residues in the $\mathrm{N}$-termini.

We found, in HvPIP2;5 overexpressing Arabidopsis, upregulation of $\mathrm{P} 5 \mathrm{CS} 2$, high levels of proline, and increased activities of SOD, CAT, GR, and APX with reduced levels of ROS under drought and salt stress conditions. Particularly, up-regulation of the key proline biosynthetic P5CS genes coincided with increased levels of osmoprotectant proline under salt stress (Figure 6). Proline mainly functions in defense and turgor pressure maintenance against water-deprived conditions (Oregan et al., 1993; Kishor et al., 1995; Khedr et al., 2003). Thus, enhanced stress tolerance in HvPIP2;5 overexpressing plants seemed to result, at least in part, from increased expression of proline biosynthetic genes and elevated activities of ROS scavenging enzymes. Similar to our findings, the overexpression of wheat TaAQP7, one of the closest homologs of $H v P I P 2 ; 5$, in tobacco enhanced drought tolerance in correlation with decreased levels of MDA and $\mathrm{H}_{2} \mathrm{O}_{2}$ and increased activities of SOD and CAT enzymes (Zhou et al., 2012). Improved osmotic stress tolerance by upregulation of stress-induced genes and an increase of ROS scavenging enzyme activity suggest that $H v P I P 2 ; 5$ overexpression might sensitize transgenic plants, making the overexpressors react faster to osmotic stress signals and eventually induce enhanced stress defense. Thus, one might speculate that HvPIP2;5 aquaporins in transgenic plants might activate osmotic and salt stress sensing or upstream steps in signaling pathways to induce better stress-tolerance mechanism than WT under stress conditions. Consistent with our speculation, it has been suggested that aquaporins may be part of an osmotic stress signaling cascade (Maurel et al., 2008); additionally, it has even been proposed that aquaporins may act as osmosensors (Hill et al., 2004). Further, study will be required to investigate this possibility.

In conclusion, we have shown that $H v P I P 2 ; 5$ can improve tolerance to salt and osmotic stresses when overexpressed in yeast and Arabidopsis. Our results contrast with a previous AtPIP2;5 overexpression study where osmotic stress sensitive phenotypes in AtPIP2;5 overexpressing plants were reported (Jang et al., 2007). These results suggest the diversity of PIP regulation and function in acquiring stress tolerance in plants. Further studies should be conducted to understand the functional differences among aquaporins for crop improvement under abiotic stress.

\section{AUTHOR CONTRIBUTIONS}

HA, BhL, and SKP designed the experiments; HA, JPA performed the experiments; GR, LS, BhL, and SKP advised the research; $\mathrm{HA}, \mathrm{BhL}$, and SKP discussed the results and wrote the paper.

\section{ACKNOWLEDGMENTS}

Authors thank the lab members for advice and help. The research was supported by Next-Generation BioGreen21 Program (PJ011006), Rural Development Administration, Republic of Korea.

\section{SUPPLEMENTARY MATERIAL}

The Supplementary Material for this article can be found online at: http://journal.frontiersin.org/article/10.3389/fpls.2016. 01566

\section{Supplementary Table 1 | Sequences of primers used in the study.}

Supplementary Figure 1 | Sequence analysis and structure prediction of HvPIP2;5 protein. (A) Alignment of full-length deduced amino acid sequences of HvPIP2;5 and its Arabidopsis homolog, AtPIP2;5. Asterisks below the alignment indicate identical amino acids. The MIPS domain is marked with a black bar below the alignment. The highly conserved amino acid sequence "HINPAVTFG" and two NPA motifs are marked with a solid-line box and a dotted-line box, respectively. The conserved phosphorylation-target serine residues in the C-termini were shown in bold. (B) Six predicted transmembrane helical regions $(\mathrm{H} 1-\mathrm{H} 6)$ were indicated with boxes in the protein sequence alignments in HvPIP2;5 and AtPIP2;5 sequences. The prediction was made by TMPred online software. (C) HvPIP2;5 secondary structure prediction using SOPMA online program. (D) Phylogenetic analysis of members of barley PIP2 clade aquaporins. GenBank accession numbers for barley PIP2 clade proteins are as follows: HvPIP2;1 (BAE02729.1), HvPIP2;2 (BAG06230.1), HvPIP2;3 (BAF33069.1), HvPIP2;4 (BAE06148.1), HvPIP2;5 (BAG06231.1), HvPIP2;7 (ADW85675.1), HvPIP2;8 (BAJ90410.1), HvPIP2;9 (BAJ92749.1), HvPIP2;10 (BAK04917.1). Neighbor-end joining (NJ) method was applied using MEGA6 with a tree file produced by Clustal omega. The scale bar indicates 0.2 substitutions per site.

Supplementary Figure 2 | Generation of HvPIP2;5 overexpressing Arabidopsis. (A) Schematic illustration of the binary vector region used for HvPIP2;5 overexpression in Arabidopsis. The complete ORF of HvPIP2;5 was cloned in pCAMBIA-2301 vector carrying CaMV35s promoter. (B) Detection of the HvPIP2:5 transcripts from the HvPIP2;5 overexpressing Arabidopsis lines by semi-quantitative RT-PCR. Ubiquitin gene was used as an internal control.

Supplementary Figure 3 | Seed germination and root growth of HvPIP2;5 overexpressing lines. $(\mathbf{A}, \mathbf{C})$ Seed germination of WT and OE lines on MS media containing $100 \mathrm{mM} \mathrm{NaCl}$ or $10 \%$ PEG. (B,D), Root elongation on WT and OE lines on MS media containing $100 \mathrm{mM} \mathrm{NaCl}$ or 10\% PEG.

Supplementary Figure 4 | Chllorophyll and relative water contents in HvPIP2;5 overexpressing lines. (A) Chlorophyll A contents under salt (100 mM) or osmotic stress (10\% PEG) conditions. (B) Relative water contents under salt (100 mM) or osmotic stress (10\% PEG) conditions. Bars represent means $\pm \mathrm{SE}$ and values with asterisks indicate significance at $P<0.05$.

Supplementary Figure 5 | Analysis of superoxide, hydrogen peroxide, malondialdehyde contents in HvPIP2;5 overexpressing lines under salt and osmotic stresses. (A) Quantification of superoxide contents in WT and OE lines after $100 \mathrm{mM} \mathrm{NaCl}$ or 10\% PEG treatments. (B) Quantification of hydrogen peroxide contents in WT and OE lines after $100 \mathrm{mM} \mathrm{NaCl}$ or $10 \%$ PEG treatments. (C) Quantification of MDA (malondialdehyde) levels in WT and OE lines after 100 $\mathrm{mM} \mathrm{NaCl}$ or $10 \% \mathrm{PEG}$ treatments. Bars indicate standard error and significant differences between WT and OE lines were marked with asterisks $(P<0.05)$.

Supplementary Figure 6 | Predicted phosphorylation sites in PIP2;5 proteins. Predicted phosphorylation sites in (A) HvPIP2;5 and (B) AtPIP2;5 (At3554820). Phosphorylation site prediction was carried out using NetPhos 2.0 (http://www.cbs.dtu.dk/services/NetPhos/). Peaks above the threshold line (red) indicate the phosphorylation sites with high probability. 


\section{REFERENCES}

Afzal, Z., Howton, T., Sun, Y., and Mukhtar, M. (2016). The roles of aquaporins in plant stress responses. J. Develop. Biol. 4:9. doi: 10.3390/jdb4010009

Aharon, R., Shahak, Y., Wininger, S., Bendov, R., Kapulnik, Y., and Galili, G. (2003). Overexpression of a plasma membrane aquaporin in transgenic tobacco improves plant vigor under favorable growth conditions but not under drought or salt stress. Plant Cell 15, 439-447. doi: 10.1105/tpc.009225

Alexandersson, E., Fraysse, L., Sjövall-Larsen, S., Gustavsson, S., Fellert, M., Karlsson, M., et al. (2005). Whole gene family expression and drought stress regulation of aquaporins. Plant Mol. Biol. 59, 469-484. doi: 10.1007/s11103005-0352-1

Bates, L. S., Waldren, R. P., and Teare, I. D. (1973). Rapid determination of free proline for water-stress studies. Plant Soil 39, 205-207. doi: 10.1007/BF00018060

Bradford, M. M. (1976). A rapid and sensitive method for the quantitation of microgram quantities of protein utilizing the principle of protein-dye binding. Anal. Biochem. 72, 248-254. doi: 10.1016/0003-2697(76)90527-3

Chance, B. A., and Maehly, A. C. (1955). "Assay of catalases and peroxidases," in Methods in Enzymology, Vol. 2, eds S. P. Colowick and N. O. Kaplan (Cambridge, MA: Academic Press), 764-775. doi: 10.1016/S00766879(55)02300-8

Chaumont, F., Barrieu, F., Jung, R., and Chrispeels, M. J. (2000). Plasma membrane intrinsic proteins from maize cluster in two sequence subgroups with differential aquaporin activity. Plant Physiol. 122, 1025-1034. doi: 10.1104/pp.122.4.1025

Chaumont, F., Barrieu, F., Wojcik, E., Chrispeels, M. J., and Jung, R. (2001). Aquaporins constitute a large and highly divergent protein family in maize. Plant Physiol. 125, 1206-1215. doi: 10.1104/pp.125.3.1206

Clough, S. J., and Bent, A. F. (1998). Floral dip: a simplified method for Agrobacterium-mediated transformation of Arabidopsis thaliana. Plant J. 16, 735-743. doi: 10.1046/j.1365-313x.1998.00343.x

Cui, X. H., Hao, F. S., Chen, H., Chen, J., and Wang, X. C. (2008). Expression of the Vicia faba VfPIP1 gene in Arabidopsis thaliana plants improves their drought resistance. J. Plant Res. 121, 207-214. doi: 10.1007/s10265-007-0130-z

Daniels, M. J., and Yeager, M. (2005). Phosphorylation of aquaporin PvTIP3;1 defined by mass spectrometry and molecular modeling. Biochemistry 44, 14443-14454. doi: 10.1021/bi050565d

Day, R. E., Kitchen, P., Owen, D. S., Bland, C., Marshall, L., Conner, A. C., et al. (2014). Human aquaporins: regulators of transcellular water flow. Biochim. Biophys. Acta Gen. Subjects 1840, 1492-1506. doi: 10.1016/j.bbagen.2013. 09.033

Diao, G. P., Wang, Y. C., Wang, C., and Yang, C. P. (2011). Cloning and functional characterization of a Novel Glutathione S-Transferase Gene from Limonium bicolor. Plant Mol. Biol. Report. 29, 77-87. doi: 10.1007/s11105-010-0212-2

Elstner, E. F., and Heupel, A. (1976). Formation of hydrogen peroxide by isolated cell walls from horseradish (Armoracia lapathifolia Gilib.). Planta 130, 175-180. doi: $10.1007 /$ BF00384416

Engel, A., and Stahlberg, H. (2002). "Aquaglyceroporins: channel proteins with a conserved core, multiple functions, and variable surfaces," in International Review of Cytology, Vol. 215, eds W. D. Stein and T. Zeuthen (Cambridge, MA: Academic Press), 75-104. doi: 10.1016/S0074-7696(02) 15006-6

Fetter, K., Van Wilder, V., Moshelion, M., and Chaumont, F. (2004). Interactions between plasma membrane aquaporins modulate their water channel activity. Plant Cell 16, 215-228. doi: 10.1105/tpc.017194

Gomes, D., Agasse, A., Thiébaud, P., Delrot, S., Gerós, H., and Chaumont, F. (2009). Aquaporins are multifunctional water and solute transporters highly divergent in living organisms. Biochim. Biophys. Acta 1788, 1213-1228. doi: 10.1016/j.bbamem.2009.03.009

Guo, L., Wang, Z. Y., Lin, H., Cui, W. E., Chen, J., Liu, M., et al. (2006). Expression and functional analysis of the rice plasma-membrane intrinsic protein gene family. Cell Res. 16, 277-286. doi: 10.1038/sj.cr.7310035

Gupta, A. S., Webb, R. P., Holaday, A. S., and Allen, R. D. (1993). Overexpression of superoxide dismutase protects plants from oxidative stress (Induction of Ascorbate Peroxidase in Superoxide Dismutase-Overexpressing plants). Plant Physiol. 103, 1067-1073.
Hayat, S., Hayat, Q., Alyemeni, M. N., Wani, A. S., Pichtel, J., and Ahmad, A. (2012). Role of proline under changing environments: a review. Plant Signal. Behav. 7, 1456-1466. doi: 10.4161/psb.21949

Heath, R. L., and Packer, L. (1968). Photoperoxidation in isolated chloroplasts. Arch. Biochem. Biophys. 125, 189-198. doi: 10.1016/0003-9861(68)90654-1

Heymann, J. B., and Engel, A. (1999). Aquaporins: phylogeny, structure, and physiology of water channels. Physiology 14, 187-193.

Hill, A. E., Shachar-Hill, B., and Shachar-Hill, Y. (2004). What are aquaporins for? J. Membr. Biol. 197, 1-32. doi: 10.1007/s00232-003-0639-6

Horie, T., Kaneko, T., Sugimoto, G., Sasano, S., Panda, S. K., Shibasaka, M., et al. (2011). Mechanisms of water transport mediated by PIP aquaporins and their regulation via phosphorylation events under salinity stress in Barley roots. Plant Cell Physiol. 52, 663-675. doi: 10.1093/pcp/pcr027

Hove, R. M., Ziemann, M., and Bhave, M. (2015). Identification and expression analysis of the Barley (Hordeum vulgare L.) Aquaporin gene family. PLoS ONE 10:e0128025. doi: 10.1371/journal.pone.0128025

Ishibashi, K., Kondo, S., Hara, S., and Morishita, Y. (2011). The evolutionary aspects of aquaporin family. Am. J. Physiol. Regul. Integr. Comparat. Physiol. 300, R566-R576. doi: 10.1152/ajpregu.90464.2008

Jahn, T. P., Moller, A. L. B., Zeuthen, T., Holm, L. M., Klaerke, D. A., Mohsin, B., et al. (2004). Aquaporin homologues in plants and mammals transport ammonia. FEBS Lett. 574, 31-36. doi: 10.1016/j.febslet.2004.08.004

Jang, J. Y., Lee, S. H., Rhee, J. Y., Chung, G. C., Ahn, S. J., and Kang, H. (2007). Transgenic Arabidopsis and tobacco plants overexpressing an aquaporin respond differently to various abiotic stresses. Plant Mol. Biol. 64, 621-632. doi: 10.1007/s11103-007-9181-8

Javot, H., Lauvergeat, V., Santoni, V., Martin-Laurent, F., Güçlü, J., Vinh, J., et al. (2003). Role of a single aquaporin isoform in root water uptake. Plant Cell 15, 509-522. doi: 10.1105/tpc.008888

Jiang, Y., Liu, H., Liu, W.-J., Tong, H.-B., Chen, C.-J., Lin, F.-G., et al. (2016). Endothelial Aquaporin-1 (AQP1) expression is regulated by transcription factor Mef2c. Mol. Cells 39, 292-298. doi: 10.14348/molcells.2016.2223

Johansson, I., Karlsson, M., Shukla, V. K., Chrispeels, M. J., Larsson, C., and Kjellbom, P. (1998). Water transport activity of the plasma membrane aquaporin PM28A is regulated by phosphorylation. Plant Cell 10, 451-459. doi: $10.1105 /$ tpc.10.3.451

Kaldenhoff, R., Grote, K., Zhu, J. J., and Zimmermann, U. (1998). Significance of plasmalemma aquaporins for water-transport in Arabidopsis thaliana. Plant J. 14, 121-128. doi: 10.1046/j.1365-313X.1998.00111.x

Katsuhara, M., Koshio, K., Shibasaka, M., Hayashi, Y., Hayakawa, T., and Kasamo, K. (2003). Over-expression of a barley aquaporin increased the shoot/root ratio and raised salt sensitivity in transgenic rice plants. Plant Cell Physiol. 44, 1378-1383. doi: 10.1093/pcp/pcg167

Katsuhara, M., Tsuji, N., Shibasaka, M., and Panda, S. K. (2014). Osmotic stress decreases PIP aquaporin transcripts in barley roots but $\mathrm{H} 2 \mathrm{O} 2$ is not involved in this process. J. Plant Res. 127, 787-792. doi: 10.1007/s10265-014-0662-y

Kawai, S., Hashimoto, W., and Murata, K. (2010). Transformation of Saccharomyces cerevisiae and other fungi: Methods and possible underlying mechanism. Bioeng. Bugs 1, 395-403. doi: 10.4161/bbug.1.6.13257

Khan, K., Agarwal, P., Shanware, A., and Sane, V. A. (2015). Heterologous expression of two Jatropha Aquaporins imparts drought and salt tolerance and improves seed viability in Transgenic Arabidopsis thaliana. PLoS ONE 10:e0128866. doi: 10.1371/journal.pone.0128866

Khedr, A. H. A., Abbas, M. A., Wahid, A. A. A., Quick, W. P., and Abogadallah, G. M. (2003). Proline induces the expression of salt-stress-responsive proteins and may improve the adaptation of Pancratium maritimum L. to salt-stress. J. Exp. Bot. 54, 2553-2562. doi: 10.1093/jxb/erg277

Kishor, P. B. K., Hong, Z., Miao, G. H., Hu, C. A. A., and Verma, D. P. S. (1995). Overexpression of Delta-Pyrroline-5-Carboxylate synthetase increases proline production and confers osmotolerance in transgenic plants. Plant Physiol. 108, 1387-1394.

Lee, H. K., Cho, S. K., Son, O., Xu, Z., Hwang, I., and Kim, W. T. (2009). Drought Stress-Induced Rma1H1, a RING Membrane-Anchor E3 Ubiquitin Ligase Homolog, Regulates Aquaporin Levels via Ubiquitination in Transgenic Arabidopsis Plants. Plant Cell 21, 622-641. doi: 10.1105/tpc.108.061994

Li, D. D., Wu, Y. J., Ruan, X. M., Li, B., Zhu, L., Wang, H., et al. (2009). Expressions of three cotton genes encoding the PIP proteins are regulated in 
root development and in response to stresses. Plant Cell Rep. 28, 291-300. doi: 10.1007/s00299-008-0626-6

Li, G., Santoni, V., and Maurel, C. (2014). Plant aquaporins: roles in plant physiology. Biochim. Biophys. Acta Gen. Subjects 1840, 1574-1582. doi: 10.1016/j.bbagen.2013.11.004

Li, J., Ban, L., Wen, H., Wang, Z., Dzyubenko, N., Chapurin, V., et al. (2015). An aquaporin protein is associated with drought stress tolerance. Biochem. Biophys. Res. Commun. 459, 208-213. doi: 10.1016/j.bbrc.2015.02.052

Lian, H.-L., Yu, X., Ye, Q., Ding, X.-S., Kitagawa, Y., Kwak, S. S., et al. (2004). The role of aquaporin RWC3 in drought avoidance in rice. Plant Cell Physiol. 45, 481-489. doi: 10.1093/pcp/pch058

Lichtenthaler, H. K. (1987). Chlorophyll Fluorescence Signatures of Leaves during the Autumnal Chlorophyll Breakdown. J. Plant Physiol. 131, 101-110. doi: 10.1016/S0176-1617(87)80271-7

Liénard, D., Durambur, G., Kiefer-Meyer, M. C., Nogué, F., Menu-Bouaouiche, L., Charlot, F., et al. (2008). Water transport by aquaporins in the extant plant Physcomitrella patens. Plant Physiol. 146, 1207-1218. doi: 10.1104/pp.107.111351

Martinez-Ballesta, M. D. C., and Carvajal, M. (2014). New challenges in plant aquaporin biotechnology. Plant Sci. 217-218, 71-77. doi: 10.1016/j.plantsci.2013.12.006

Martre, P., Morillon, R., Barrieu, F., North, G. B., Nobel, P. S., and Chrispeels, M. J. (2002). Plasma membrane Aquaporins play a significant role during recovery from water deficit. Plant Physiol. 130, 2101-2110. doi: 10.1104/pp.009019

Maurel, C. (2007). Plant aquaporins: novel functions and regulation properties. FEBS Lett. 581, 2227-2236. doi: 10.1016/j.febslet.2007.03.021

Maurel, C., Boursiac, Y., Luu, D. T., Santoni, V., Shahzad, Z., and Verdoucq, L. (2015). Aquaporins in plants. Physiol. Rev. 95, 1321-1358. doi: 10.1152/physrev.00008.2015

Maurel, C., Verdoucq, L., Luu, D. T., and Santoni, V. (2008). Plant aquaporins: membrane channels with multiple integrated functions. Annu. Rev. Plant Biol. 59, 595-624. doi: 10.1146/annurev.arplant.59.032607.092734

Nakano, Y., and Asada, K. (1981). Hydrogen Peroxide is Scavenged by Ascorbatespecific Peroxidase in Spinach Chloroplasts. Plant Cell Physiol. 22, 867-880.

Oregan, B. P., Cress, W. A., and Vanstaden, J. (1993). Root-growth, water relations, abscisic-acid and proline levels of drought-resistant and drought-sensitive maize cultivars in response to water-stress. South Afr. J. Bot. 59, 98-104. doi: 10.1016/S0254-6299(16)30780-3

Pang, C.-H., and Wang, B.-S. (2010). "Role of ascorbate peroxidase and glutathione reductase in Ascorbate-Glutathione cycle and stress tolerance in plants," in Ascorbate-Glutathione Pathway and Stress Tolerance in Plants, eds A. N. Anjum, M.-T. Chan, and S. Umar (Dordrecht: Springer), 91-113.

Park, J. H., and Saier, M. H. Jr. (1996). Phylogenetic characterization of the MIP family of transmembrane channel proteins. J. Membrane Biol. 153, 171-180. doi: $10.1007 /$ s002329900120

Postaire, O., Tournaire-Roux, C., Grondin, A., Boursiac, Y., Morillon, R., Schäffner, A. R., et al. (2010). A PIP1 aquaporin contributes to hydrostatic pressure-induced water transport in both the root and rosette of Arabidopsis. Plant Physiol. 152, 1418-1430. doi: 10.1104/pp.109.145326

Ramel, F., Sulmon, C., Bogard, M., Couée, I., and Gouesbet, G. (2009). Differential patterns of reactive oxygen species and antioxidative mechanisms during atrazine injury and sucrose-induced tolerance in Arabidopsis thaliana plantlets. BMC Plant Biol. 9:28. doi: 10.1186/1471-2229-9-28

Rao, M. V., and Davis, K. R. (1999). Ozone-induced cell death occurs via two distinct mechanisms in Arabidopsis: the role of salicylic acid. Plant J. 17, 603-614. doi: 10.1046/j.1365-313X.1999.00400.x

Sade, N., Gebretsadik, M., Seligmann, R., Schwartz, A., Wallach, R., and Moshelion, M. (2010). The role of tobacco aquaporin1 in improving water use efficiency, hydraulic conductivity, and yield production under salt stress. Plant Physiol. 152, 245-254. doi: 10.1104/pp.109.145854
Sagisaka, S. (1976). The Occurrence of Peroxide in a Perennial Plant, Populus gelrica. Plant Physiol. 57, 308-309. doi: 10.1104/pp.57.2.308

Santoni, V., Vinh, J., Pflieger, D., Sommerer, N., and Maurel, C. (2003). A proteomic study reveals novel insights into the diversity of aquaporin forms expressed in the plasma membrane of plant roots. Biochem. J. 373, 289-296. doi: 10.1042/bj20030159

Siefritz, F., Tyree, M. T., Lovisolo, C., Schubert, A., and Kaldenhoff, R. (2002). PIP1 plasma membrane aquaporins in tobacco: from cellular effects to function in plants. Plant Cell 14, 869-876. doi: 10.1105/tpc.000901

Smith, I. K., Vierheller, T. L., and Thorne, C. A. (1988). Assay of glutathione reductase in crude tissue homogenates using 5,5'-dithiobis(2nitrobenzoic acid). Anal. Biochem. 175: 408-413. doi: 10.1016/0003-2697(88) 90564-7

Spring, J. H., Robichaux, S. R., and Hamlin, J. A. (2009). The role of aquaporins in excretion in insects. J. Exp. Biol. 212, 358-362. doi: 10.1242/jeb.024794

Sun, L., Yu, G., Han, X., Xin, S., Qiang, X., Jiang, L., et al. (2015). TsMIP6 enhances the tolerance of transgenic rice to salt stress and interacts with target proteins. J. Plant Biol. 58, 285-292. doi: 10.1007/s12374-015-0069-x

Uehlein, N., Lovisolo, C., Siefritz, F., and Kaldenhoff, R. (2003). The tobacco aquaporin NtAQP1 is a membrane $\mathrm{CO}_{2}$ pore with physiological functions. Nature 425, 734-737. doi: 10.1038/nature02027

Walz, T., Hirai, T., Murata, K., Heymann, J. B., Mitsuoka, K., Fujiyoshi, Y., et al. (1997). The three-dimensional structure of aquaporin-1. Nature 387, 624-627. doi: $10.1038 / 42512$

Wang, L. L., Chen, A. P., Zhong, N. Q., Liu, N., Wu, X. M., Wang, F., et al. (2014). The Thellungiella salsuginea tonoplast aquaporin TsTIP1;2 functions in protection against multiple abiotic stresses. Plant Cell Physiol. 55, 148-161. doi: $10.1093 / \mathrm{pcp} / \mathrm{pct} 166$

Weatherley, P. E. (1950). Studies in the water relations of the cotton plant. New Phytol. 49, 81-97. doi: 10.1111/j.1469-8137.1950.tb05146.x

Zardoya, R. (2005). Phylogeny and evolution of the major intrinsic protein family. Biol. Cell 97, 397-414. doi: 10.1042/BC20040134

Zardoya, R., Ding, X., Kitagawa, Y., and Chrispeels, M. J. (2002). Origin of plant glycerol transporters by horizontal gene transfer and functional recruitment. Proc. Natl. Acad. Sci. U.S.A. 99, 14893-14896. doi: 10.1073/pnas.1925 73799

Zelazny, E., Borst, J. W., Muylaert, M., Batoko, H., Hemminga, M. A., and Chaumont, F. (2007). FRET imaging in living maize cells reveals that plasma membrane aquaporins interact to regulate their subcellular localization. Proc. Natl. Acad. Sci. U.S.A. 104, 12359-12364. doi: 10.1073/pnas.0701180104

Zhou, L., Wang, C., Liu, R., Han, Q., Vandeleur, R. K., Du, J., et al. (2014). Constitutive overexpression of soybean plasma membrane intrinsic protein GmPIP1;6 confers salt tolerance. BMC Plant Biol. 14:181. doi: 10.1186/14712229-14-181

Zhou, S., Hu, W., Deng, X., Ma, Z., Chen, L., Huang, C., et al. (2012). Overexpression of the wheat aquaporin gene, TaAQP7, enhances drought tolerance in Transgenic Tobacco. PLoS ONE 7:e52439. doi: 10.1371/journal.pone.0052439

Conflict of Interest Statement: The authors declare that the research was conducted in the absence of any commercial or financial relationships that could be construed as a potential conflict of interest.

Copyright (c) 2016 Alavilli, Awasthi, Rout, Sahoo, Lee and Panda. This is an openaccess article distributed under the terms of the Creative Commons Attribution License (CC BY). The use, distribution or reproduction in other forums is permitted, provided the original author(s) or licensor are credited and that the original publication in this journal is cited, in accordance with accepted academic practice. No use, distribution or reproduction is permitted which does not comply with these terms. 